\title{
Texture analysis of diffusion weighted imaging for the evaluation of glioma heterogeneity based on different regions of interest
}

\author{
SHAN WANG ${ }^{1,2^{*}}$, MENG MENG $^{3 *}$, XUE ZHANG $^{1}$, CHEN WU $^{1}$, RU WANG $^{1}$, \\ JIANGFEN WU ${ }^{4}$, MUHAMMAD UMAIR SAMI ${ }^{1}$ and KAI XU ${ }^{1,5}$

\begin{abstract}
${ }^{1}$ School of Medical Imaging, Xuzhou Medical University, Xuzhou, Jiangsu 221004; ${ }^{2}$ Department of Radiology, Jiangsu Jiangyin People's Hospital, Jiangyin, Jiangsu 214400; ${ }^{3}$ School of Medical Imaging, Guizhou Medical University, Guiyang, Guizhou 550004; ${ }^{4}$ GE Healthcare (Shanghai) Co., Ltd., Shanghai 201203;
\end{abstract} \\ ${ }^{5}$ Department of Radiology, Affiliated Hospital of Xuzhou Medical University, Xuzhou, Jiangsu 221006, P.R. China
}

Received May 17, 2017; Accepted February 23, 2018

DOI: $10.3892 /$ ol.2018.8232

\begin{abstract}
The present study aimed to explore the role of texture analysis with apparent diffusion coefficient (ADC) maps based on different regions of interest (ROI) in determining glioma grade. Thirty patients with glioma underwent diffusion-weighted imaging (DWI). ADC values were determined from the following three ROIs: i) whole tumor; ii) solid portion; and iii) peritumoral edema. Texture features were compared between high-grade gliomas (HGGs) and low-grade gliomas (LGGs) using the non-parametric Wilcoxon rank-sum test or the unpaired Student's t-test. Receiver operating characteristic (ROC) curves were constructed to determine the optimum threshold for inhomogeneity values in discrimination of HGGs from LGGs. With a spearman rank correlation model, the aforementioned ADC inhomogeneity values were correlated with the Ki-67 labeling index. With whole tumor ROI, inhomogeneity values proved to be significantly different between HGGs and LGGs $(\mathrm{P}<0.001)$. With solid portion ROI, inhomogeneity and median values showed significant difference between HGGs and LGGs ( $\mathrm{P}=0.001$ and $\mathrm{P}=0.043$, respectively). With peritumoral edema $\mathrm{ROI}$, entropy and edema volume demonstrated positive results $(\mathrm{P}=0.016, \mathrm{P}<0.001)$. The whole tumor inhomogeneity parameter performed with better diagnostic accuracy $(\mathrm{P}=0.048)$ than selecting the solid portion ROI. The association between inhomogeneity and Ki-67 labeling index was significantly positive in whole tumor and solid portion $\mathrm{ROI}(\mathrm{R}=0.628, \mathrm{P}<0.001$ and $\mathrm{R}=0.470, \mathrm{P}=0.009)$. Texture analysis of DWI based on different ROI can provide
\end{abstract}

Correspondence to: Professor Kai Xu, School of Medical Imaging, Xuzhou Medical University, 209 Tongshan Road, Xuzhou, Jiangsu 221004, P.R. China

E-mail: xkpaper@163.com

${ }^{*}$ Contributed equally

Key words: glioma, grading, diffusion-weighted imaging, texture analysis, region of interest various significant parameters to evaluate tumor heterogeneity, which were correlated with tumor grade. Particularly, the inhomogeneity value derived from whole tumor ROI provided high diagnostic value and predicting the status of tumor proliferation.

\section{Introduction}

Gliomas are the most common type of primary brain tumor. The World Health Organization (WHO) classifies gliomas into grades I-IV, where I and II are low-grade gliomas (LGGs) and III and IV are high-grade gliomas (HGGs) (1). Determining the correct grade of the tumor is of great importance as it dictates the management and prognosis for the patient (2). HGGs are managed with radical resection and with adjuvant radiotherapy and/or chemotherapy, whereas LGGs are very slow growing and can undergo curative resection and have considerably better prognosis (3). The current gold standard for grading gliomas is histopathological assessment by stereotactic brain biopsy, which is an invasive procedure. Particularly with gliomas, the potential to increase clinical utility of imaging as a non-invasive technique to accurately ascertain tumor grade is gaining a lot of attention (4).

Advanced MR imaging techniques such as diffusion-weighted imaging (DWI) and its estimated apparent diffusion coefficient (ADC) can probe the pathological changes in glioma providing abundant important information that is not apparent on conventional imaging (5-7). ADC, which reflects the volume of the extracellular water compartment, is sensitive to changes in cell density, edema, and necrosis (8-10). Mostradiologists estimate tumor classification via simple visual inspection of ADC, however, this inevitably has a lot of subjective factors. The method of texture analysis that quantifies tumor heterogeneity on routinely available images could offer a complementary tool to existing radiological practice in differentiating HGGs from LGGs (2).

Texture analysis is a method for quantifying the spatial distributions of intensities in images. It has shown promise in the field of oncology diagnosis $(11,12)$, quantifying tumor heterogeneity (13), separating tumor tissue from surrounding tissue $(14,15)$, tumor grading and classification (16-18), and 
predictions of treatment response and survival $(19,20)$. Until now, some reports have been published regarding tumor heterogeneity in intracranial tumors using CT and MRI texture analysis. However, the choice of region of interest is variable. A study performed by Skogen et al (2) only drew the area within the contrast enhanced tumor and excluded peritumoral edema for CT texture analysis in quantifying tumor heterogeneity of gliomas and showed a correlation between tumor heterogeneity and tumor grade. Skogen et al (21) delineated the solid tumor for the regions of interest (ROI), and revealed that textural features extracted on contrast-enhanced (CE) images were highly discriminant between LGG and HGG. Conversely, Kang et al (22) choose whole tumor for analysis, to account for all elements of the tumor that could contribute to group differences. It is widely known that glioma is the most complicated tumor in the central nervous system. The definition of ROI had a great influence on the analysis results in each of these studies. Therefore, we divided gliomas into three parts: i) solid portion; ii) peritumoral edema; and iii) the others (like necrosis and hemorrhage). ADC values of solid portion, peritumoral edema and whole tumor (which contains all elements of the tumor) were determined.

The aim of this study was to apply texture analysis of ADC maps in order to explore the significant parameters in different ROI to evaluate tumor heterogeneity, which was correlated with tumor grade and to compare the diagnostic performance of different ROI furtherly.

\section{Materials and methods}

Patient selection. Sixty-four patients (26 women and 38 men, mean age $47.6 \pm 15.1$ years) with astrocytic tumors who had underwent initial MR imaging at the affiliated hospital of Xuzhou medical university between October 2010 and January 2016 were selected from the radiology report database. Inclusion criteria were as follows: i) histopathological diagnosis of gliomas according to the WHO criteria; ii) MR imaging performed with DWI at the standard $b$ value prior to surgery or chemoradiotherapy. Thirty-four patients were excluded, due to the following reasons: i) inadequate MR imaging quality due to substantial motion or susceptibility artifacts ( $\mathrm{n}=7)$; ii) MR imaging performed at $1.5 \mathrm{~T}(\mathrm{n}=22)$; iii) small size (maximum diameter $<1 \mathrm{~cm}$ ) of the tumor $(\mathrm{n}=2)$; and iv) absence of $\mathrm{T} 1$ contrast enhanced images, which provided basis for the division of different tumor areas $(n=3)$. Finally, 30 patients were included in this study. A total of 12 patients exhibited LGGs (astrocytomas), 18 patients exhibited HGGs (9 anaplastic astrocytomas and 9 glioblastomas) according to the classification of WHO (Table I). The present study was approved by the Ethical Committee of the First Affiliated Hospital of Xuzhou Medical University, Xuzhou, China. All patients provided written informed consent for participation in the present study.

MR imaging. All patients were examined with the same imaging acquisition protocol on a $3.0 \mathrm{~T}$ whole-body MRI system [Signa HD; GE Healthcare (Shanghai) Co., Ltd., Shanghai, China]. The MRI protocol consisted of a T1 inversion recovery (T1IR) sequence with the following parameters: TR/TE/TI 2150/15/820 ms, FOV 240x240 mm, slice thickness
$6 \mathrm{~mm}$, and slice gap $1.5 \mathrm{~mm}$. The T2-weighted sequence was performed with TR/TE 4,300/120 ms, FOV 240x240 mm, slice thickness $6 \mathrm{~mm}$, slice gap $1.5 \mathrm{~mm}$. The fluid-attenuated inversion-recovery (FLAIR) sequence was performed with TR/TE/TI 9600/155/2250 ms, FOV 240x240 mm, slice thickness $6 \mathrm{~mm}$, slice gap $1.5 \mathrm{~mm}$. DWI $\left(b=0\right.$ and $1,000 \mathrm{sec} / \mathrm{mm}^{2}$; 18 sections; section thickness $6 \mathrm{~mm}$; intersection gap $1.5 \mathrm{~mm}$; field of view 240×240 mm) was performed in the axial plane. Contrast agent [Omniscan; GE Healthcare (Shanghai) Co., Ltd.] was administered at rate of $2 \mathrm{ml} / \mathrm{sec}$ for a $\mathrm{T} 1$ enhanced sequence, with a dose of $0.2 \mathrm{ml} / \mathrm{kg}$.

Volume acquisition. MR data were digitally transferred from the PACS workstation to a personal computer, and two-dimensional (2-D) ROI were drawn in each slice of DWI using software. The data acquired from each slice was summated to derive voxel-by-voxel ADCs for the entire tumor, which guaranteed a set of relatively complete three-dimensional (3-D) information of the tumor. Glioma lesions were divided into three parts on DWI: i) Solid portion; ii) peritumoral edema area; and iii) the others respectively. Then the whole tumor, solid portion and peritumoral edema ROIs were drawn by the same researcher with guidance of an experienced neuroradiologist, without knowledge of the final histological tumor diagnosis. Whole tumor boundaries were defined with reference to the high signal intensity areas thought to represent tumor tissue on the T2WI by one author (a neuroradiologist with thirty years of brain MR imaging experience) via visual inspection (23). The solid portions were deliniated from the whole tumor excluding peritumoral edema, definite cystic, necrotic, and hemorrhagic areas. For visual inspection, we used strict criteria of 'definite' necrosis as a nonenhanced portion on CE T1-weighted image and similar signal intensity to CSF on T2-weighted and FLAIR images. We did not define necrosis as nonenhanced portions, as is typically done, because $14-45 \%$ of non-enhanced supratentorial gliomas are malignant, and $25-31 \%$ of GBMs showed faint or no detectable enhancement (24) because contrast enhancement on conventional MRI only means the disruption of the blood-brain barrier, not neovascularization (25) (Fig. 3).

Texture analysis. To quantify image texture features within the ROIs, Fire voxel (https://wp.nyu.edu/firevoxel/) software was used for quantification of heterogeneous signal. Histogram analysis was utilized to calculate the first order parameters. Texture analysis via Gray Level Co-occurrence Matrices (GLCM) was implemented to extract second order features from the images. In this study, texture analysis was performed within 3-D ROI, and 4 parameters were used for the quantitative analysis including entropy, skewness, kurtosis and inhomogeneity.

$$
\begin{aligned}
& \text { Entropy }=-\sum_{\mathrm{i}=0}^{\mathrm{G}-1} \sum_{j=0}^{G-1} \mathrm{p}(i, j) \times \log (\mathrm{p}(\mathrm{i}, \mathrm{j})) \\
& \text { Inhomogeneity }=\frac{\sqrt{\frac{1}{n-1} \sum_{(x, y \in R)}[\boldsymbol{a}(\boldsymbol{x}, \boldsymbol{y})-\overline{\boldsymbol{a}}]^{2}}}{\overline{\boldsymbol{a}}}
\end{aligned}
$$

In equation (a), $G$ is the number of gray levels used. $P_{x}(i)$ is the $i_{t h}$ entry in the marginal-probability matrix obtained 
Table I. Characteristics of inclusion lesion.

\begin{tabular}{lcc}
\hline Lesion & Low grade (II) & High grade (III and IV) \\
\hline Total & 12 & 18 \\
Edema & 7 & 18 \\
Hemorrhage & 0 & 2 \\
Cystic change & 1 & 10 \\
\hline
\end{tabular}

by summing the rows of $\mathrm{P}(\mathrm{i}, \mathrm{j})$. Higher entropy represents increased heterogeneity. In equation (b), R is the ROI within the image a $(x, y), n$ is the total number of pixels in $R$ and $\bar{\alpha}$ is the mean value within R. Higher inhomogeneity means higher average variation of entire gray levels, which also represents increased heterogeneity.

Histopathological analysis. The tissue samples were obtained via image-guided tissue biopsy. Immunohistochemistry was used to measure the Ki-67 labeling index. The routinely used formalin-fixed, paraffin-embedded tissue blocks were sectioned into 4-mm thick slices and then used for immunohistochemistry. The areas with the highest cellularity on inspection were selected, and the Ki-67 labeling index was evaluated using the avidin-biotin complex immunohistochemical technique.

Statistical analysis. To compare kurtosis and inhomogeneity parameters between HGGs and LGGs, non-parametric Wilcoxon rank-sum test was applied. Statistical comparisons of the other parameters between HGGs and LGGs were performed with the independent sample t-test. For the parameters that had statistically significant differences between HGGs and LGGs, a one-way analysis of variance with Tukey's post hoc analysis or k-dependent samples of non-parametric tests were performed according to the distribution of data types to compare each grade. For some parameters that correlated significantly with tumor grade, ROCs analysis was used to characterize the diagnostic performance. The McNemar test was then performed to compare the diagnostic accuracies of different ROIs. With a spearman rank correlation model, the ADC inhomogeneity values described above were correlated with the Ki-67 labeling index. $\mathrm{P}<0.05$ was considered to indicate a statistically significant difference.

\section{Results}

Texture analysis based on each ROI between HGGs and $L G G s$. The results of texture analysis based on whole tumor and solid portion ROI revealed that the inhomogenity value were significantly different between HGGs and LGGs $(\mathrm{P}<0.001, \mathrm{P}=0.001$; Tables II and III). Additionally, lower ADC median was observed in high grade gliomas compared to low grade tumors $(\mathrm{P}=0.043)$ in solid portion $\mathrm{ROI}$.

With reference to peritumoral edema, the entropy value and edema volume were observed to be significantly higher in HGGs than LGGs ( $\mathrm{P}=0.016, \mathrm{P}<0.001$, Table IV, respectively).

Texture analysis based on whole tumor and solid portion ROI among each grade glioma. For whole tumor ROI, the
Table II. The whole tumor ADC histogram parameters of low and high grade gliomas.

\begin{tabular}{lccr}
\hline Parameter & $\begin{array}{c}\text { Low grade } \\
(\mathrm{n}=12)\end{array}$ & $\begin{array}{c}\text { High grade } \\
(\mathrm{n}=18)\end{array}$ & P-value \\
\hline Texture parameter & & & \\
$\quad$ Entropy & $5.84 \pm 0.24$ & $5.70 \pm 0.34$ & 0.218 \\
Skewness & $0.76 \pm 0.30$ & $0.66 \pm 0.41$ & 0.477 \\
Kurtosis & $1.53 \pm 1.93$ & $1.72 \pm 1.28$ & 0.189 \\
Inhomogeneity & $0.18 \pm 0.04$ & $0.32 \pm 0.09$ & $<0.001$ \\
ADC $\left(\times 10^{-3} \mathrm{~mm}^{2} / \mathrm{sec}\right)$ & & & \\
Median & $1.38 \pm 0.24$ & $1.44 \pm 0.51$ & 0.751 \\
Mean & $1.38 \pm 0.21$ & $1.25 \pm 0.38$ & 0.529 \\
\hline
\end{tabular}

ADC, apparent diffusion coefficient.

Table III. The solid portion ADC histogram parameters of low and high grade gliomas.

\begin{tabular}{lccc}
\hline Parameter & $\begin{array}{c}\text { Low grade } \\
(\mathrm{n}=12)\end{array}$ & $\begin{array}{c}\text { High grade } \\
(\mathrm{n}=18)\end{array}$ & P-value \\
\hline $\begin{array}{l}\text { Texture parameter } \\
\quad \text { Entropy }\end{array}$ & $5.86 \pm 0.21$ & $5.68 \pm 0.30$ & 0.077 \\
$\quad$ Skewness & $0.73 \pm 0.40$ & $0.85 \pm 0.56$ & 0.527 \\
Kurtosis & $1.20 \pm 1.52$ & $2.52 \pm 2.39$ & 0.352 \\
Inhomogeneity & $0.19 \pm 0.05$ & $0.29 \pm 0.09$ & 0.001 \\
ADC $\left(x 10^{-3} \mathrm{~mm}^{2} / \mathrm{sec}\right)$ & & & \\
$\quad$ Median & $1.37 \pm 0.25$ & $1.19 \pm 0.21$ & 0.043 \\
Mean & $1.37 \pm 0.22$ & $1.25 \pm 0.23$ & 0.179 \\
\hline
\end{tabular}

ADC, apparent diffusion coefficient.

Table IV. The peritumoral edema ADC histogram parameters of low and high grade gliomas.

\begin{tabular}{lccr}
\hline Parameter & $\begin{array}{c}\text { Low grade } \\
(\mathrm{n}=7)\end{array}$ & $\begin{array}{c}\text { High grade } \\
(\mathrm{n}=18)\end{array}$ & P-value \\
\hline Texture parameter & & & \\
$\quad$ Entropy & $5.87 \pm 0.22$ & $6.08 \pm 0.10$ & 0.016 \\
Skewness & $0.38 \pm 0.26$ & $0.41 \pm 0.30$ & 0.800 \\
Kurtosis & $0.49 \pm 0.22$ & $0.61 \pm 0.35$ & 0.392 \\
Inhomogeneity & $0.19 \pm 0.04$ & $0.22 \pm 0.04$ & 0.132 \\
ADC $\left(\mathrm{x} 10^{-3} \mathrm{~mm}^{2} / \mathrm{sec}\right)$ & & & \\
Median & $1.37 \pm 0.14$ & $1.40 \pm 0.25$ & 0.865 \\
Mean & $1.37 \pm 0.12$ & $1.39 \pm 0.22$ & 0.952 \\
Edema volume $\left(\mathrm{cm}^{3}\right)$ & $6.36 \pm 3.19$ & $28.74 \pm 21.20$ & $<0.001$ \\
& & &
\end{tabular}

ADC, apparent diffusion coefficient.

inhomogeneity value differed significantly between grades II and IV $(\mathrm{P}=0.01)$ and between grades II and III $(\mathrm{P}=0.005)$, but 
Table V. Histogram parameters of different ROIs among the grade II, III and IV gliomas.

P-value

\begin{tabular}{lccccc} 
Parameter & Grade II $(\mathrm{n}=11)$ & Grade III $(\mathrm{n}=9)$ & Grade IV $(\mathrm{n}=9)$ & II vs. III & II vs. IV III vs. IV \\
\hline $\begin{array}{l}\text { ROI's inhomogeneity } \\
\text { Whole tumor }\end{array}$ & $0.184 \pm 0.036$ & $0.337 \pm 0.102$ & $0.294 \pm 0.083$ & 0.005 & 0.010 \\
Solid portion & $0.185 \pm 0.051$ & $0.288 \pm 0.076$ & $0.288 \pm 0.107$ & 0.010 & 0.056 \\
$\begin{array}{l}\text { ROI's median } \\
\text { Whole tumor }\end{array}$ & $1.380 \pm 0.238$ & $1.271 \pm 0.266$ & $1.600 \pm 0.644$ & 0.236 & 0.987 \\
Solid tumor & $1.368 \pm 0.252$ & $1.172 \pm 0.245$ & $1.200 \pm 0.191$ & 0.129 \\
\hline
\end{tabular}

ROI, regions of interest.

Table VI. Different ROI's ROC results for inhomogeneity of ADC for glioma grading (high vs. low).

\begin{tabular}{lcc}
\hline & \multicolumn{2}{c}{ ROI's inhomogeneity } \\
\cline { 2 - 3 } Characteristic & Whole tumor & Solid portion \\
\hline AUC & $0.968(0.901,0.998)$ & $0.852(0.809,0.895)$ \\
Sensitivity (\%) & $0.944(17 / 18)$ & $0.833(15 / 18)$ \\
Specificity (\%) & $0.916(11 / 12)$ & $0.833(10 / 12)$ \\
Accuracy (\%) & $0.933(28 / 30)$ & $0.833(25 / 30)$ \\
Cut off value & $>0.216$ & $>0.202$ \\
P-value for & $<0.001$ & 0.001 \\
ROC curve & & \\
\hline
\end{tabular}

ROI, regions of interest; ADC, apparent diffusion coefficient; ROC, receiver operating characteristic.

not between grades III and IV (Table V). Conversely, for solid portion ROI, a significant difference was found only between grades II and III $(\mathrm{P}=0.01)$. However, no significant difference was found in the ADC median both in whole tumor ROI and solid-portion ROI among the three grades. $(\mathrm{P}=0.236$ and $\mathrm{P}=0.129$ respectively).

Diagnostic efficiency of inhomogeneity value based on whole tumor and solid portion ROI. Table VI summarized the receiver operating characteristic (ROC) results of different ROI inhomogeneity values used to distinguish HGGs from LGGs. In terms of whole tumor ROI, the inhomogeneity cutoff value of 0.2162 exhibited a sensitivity, specificity and accuracy of 94.4, 91.6 and $93.3 \%$, respectively. For solid portion ROI, the inhomogeneity cutoff value of 0.2015 exhibited a sensitivity, specificity and accuracy of 83.3, 83.3 and $83.3 \%$, respectively (Table VI, Fig. 1). There was significant difference between the diagnostic accuracy of different ROI inhomogeneity in discrimination of HGGs from LGGs ( $\mathrm{P}=0.048)$. For differentiating HGGs from LGGs, the greatest area under the curve (AUC) was observed in whole tumor ROI texture analysis $(0.968, \mathrm{P}<0.001)$.

The correlation study of inhomogeneity value and $\mathrm{Ki}-67$ labeling index. There was a significantly positive relationship

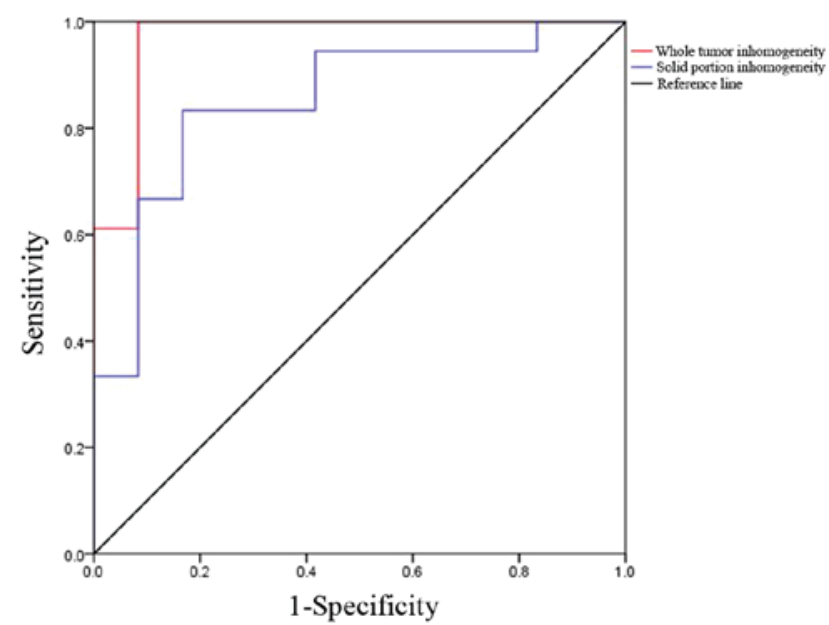

Figure 1. ROC curves for inhomogeneity of glioma grading (high vs. low). ROC, receiver operating characteristic.

between inhomogeneity values and $\mathrm{Ki}-67$ labeling index both in whole tumor and solid portion ROI $(\mathrm{R}=0.628, \mathrm{P}<0.001$; $\mathrm{R}=0.470, \mathrm{P}=0.009$ ) (Fig. 2).

Images with each ROI delineation. Fig. 3 shows one slice of representative T2-weighted images and DWIs with whole tumor, solid portion, and peritumoral edema ROI delineation for each grade of glioma. The three rows from top to bottom correspond to grade IV, III and II gliomas, respectively. The first line shows T2-weighted images of each grade glioma, The second line shows whole tumor ROIs delineation on DWI for each grade glioma. The third line shows solid portion ROIs delineation on DWI for each grade glioma. The last line shows peritumoral edema ROIs delineation on DWI for each grade glioma.

Histograms and cumulative histograms of each grade glioma based on each ROI. Fig. 4 show histograms and the corresponding cumulative histograms of each grade gliomas in whole-tumor, solid-portion and peritumoral edema ROI. Grade IV glioma showed a wider spectrum of the ADC signal intensity, exhibited a distinctly asymmetric distribution and had a high ADC inhomogeneity value $(0.44,0.42)$ in whole 
A

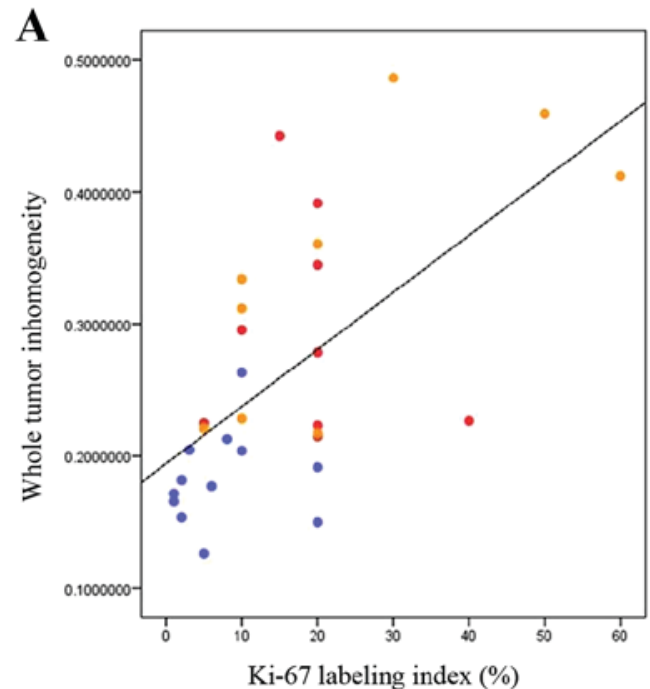

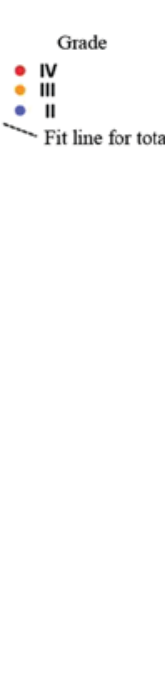

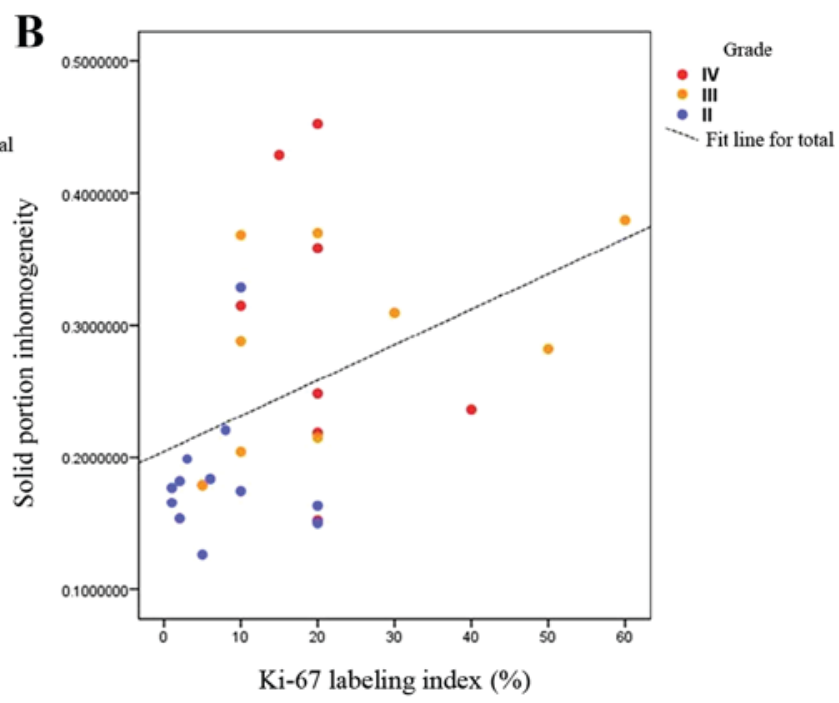

Figure 2. The relationship between inhomogeneity value and Ki-67 labeling index labeling in (A) whole tumor and (B) solid portion ROI. ROI, regions of interest.

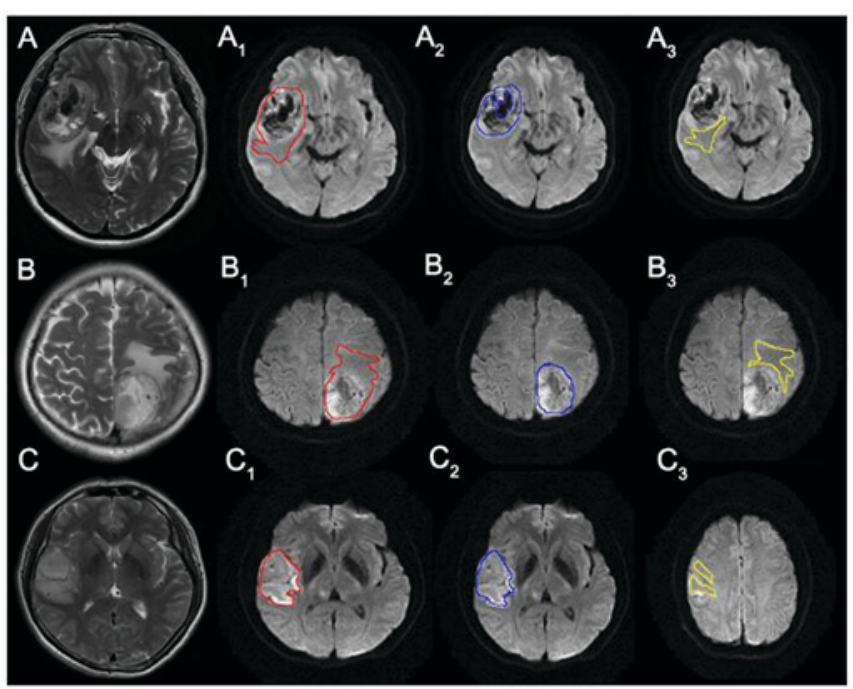

Figure 3. Rows from top to bottom are images of grade IV, III and II glioma. Line 1: T2-weighted images for (A) grade IV, (B) grade III and (C) grade II Line 2: DWI with whole tumor ROI placement for $\left(\mathrm{A}_{1}\right)$ grade IV, $\left(\mathrm{B}_{1}\right)$ grade III and $\left(\mathrm{C}_{1}\right)$ grade II. Line 3: DWI with solid portion ROI placement for $\left(A_{2}\right)$ grade IV, $\left(B_{2}\right)$ grade III and $\left(C_{2}\right)$ grade II. Line 4: DWI with peritumoral edema ROI placement for $\left(\mathrm{A}_{3}\right)$ grade IV, $\left(\mathrm{B}_{3}\right)$ grade III and $\left(\mathrm{C}_{3}\right)$ grade II.

tumor ROI and solid portion ROI (Fig. 4A and C). In contrast, grade II glioma displayed a narrower spectrum of the ADC signal intensity, exhibited a relatively symmetric distribution and had a low ADC inhomogeneity value $(0.21,0.22)$ in whole tumor ROI and solid portion ROI (Fig. 4A and C). ADC histograms of high grade glioma (Fig. 4A and C) showed a higher relative frequency at the low ADCs compared with grade II glioma, resulting in substantial divergence between low and high grade glioma at the low end of the cumulative histograms (Fig. 4B and D). This suggests that the high grade glioma contained more pixels with low ADCs, which indicates high cellularity. In terms of peritumoral edema ROI, Grade IV glioma also exhibited more asymmetrical distribution than low grade glioma and had a higher ADC entropy value (6.079 vs. 5.64) than low grade glioma (Fig. 4E).

\section{Discussion}

The results of our study suggest that texture analysis parameters for inhomogeneity or entropy based on the three ROIs could be used to differentiate HGGs from LGGs, as higher inhomogeneity or entropy was demonstrated in higher grades. However, the traditional statistics (mean and median ADC) appeared to have limited value in distinguishing tumor grade. Whole tumor inhomogeneity value may be useful in discriminating between grades II and IV and between grades II and III. Solid portion inhomogeneity value was better for discriminating between grades II and III. Inhomogeneity value seemed to be the overall best parameter in differentiating each grade. And furthermore in terms of inhomogeneity parameter, whole tumor ROI presented a better diagnostic performance than solid portion ROI in distinguishing HGGs from LGGs.

Texture analysis parameters are statistically better than traditional methods of tumor grading. In previous studies, the traditional statistics such as mean median or minimum of ADC was used to distinguish HGGs and LGGs $(22,26)$. However, these feature values reflect only a small portion of the tumor. Instead, texture analysis parameters, such as inhomogeneity and entropy, represent characteristics of the entire tumor and have the advantage of noninvasively quantifying tumor heterogeneity. Tumors are heterogeneous both genetically and histopathologically, with intratumoral spatial variation in the cellularity, angiogenesis, extravascular extracellular matrix, and areas of necrosis (27). Thus, we postulated that the heterogeneity of ADC values within gliomas can be useful for distinguishing each grade. The inhomogeneity value determined by texture analysis, represents the width of the histogram or degree of average variation from the mean pixel value. Skogen et al (21) has proposed that histogram statistical parameter standard deviation (SD) can provide useful information quantifying glioma heterogeneity on CE images. Inhomogeneity in our study was similar to $\mathrm{SD}$, but more objective, because it includes an average ADC values in 

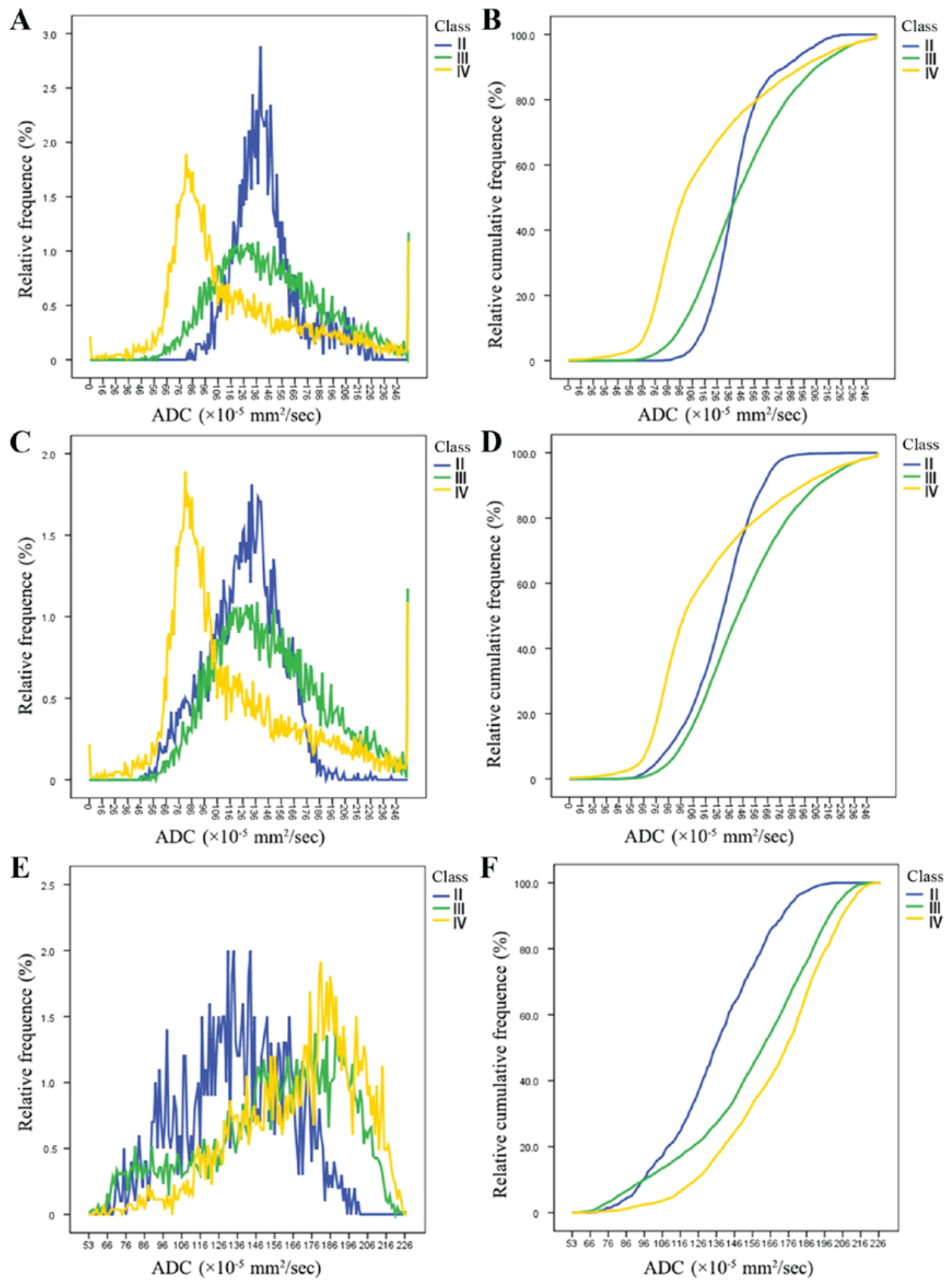

Figure 4. (A) ADC histograms and (B) its corresponding cumulative histograms of each grade glioma obtained from whole tumor ROI. (C) ADC histograms and (D) its corresponding cumulative histograms for each grade glioma obtained from solid portion ROI. (E) ADC histograms and (F) its corresponding cumulative histograms for each grade glioma obtained from peritumoral edema ROI. ROI, regions of interest; ADC, apparent diffusion coefficient.

the calculation which excluded the average level of different objects. Inhomogeneity parameters emphasize the different heterogeneous appearances of LGGs and HGGs as well as between glioma subgroups on DWI images. In this study conventional statistic median implied positive predicion only in solid portion as a lower median in HGGs. but the median data appeared to have no value in distinguishing each grade. Higher ADC values in intracranial tumors are attributed to low tumor cellularity, necrosis, or cysts, and lower values are attributed to high tumor cellularity (28). To exclude the necrosis or cysts, we analyzed the solid portions of gliomas and concluded the median ADC values were lower in higher grades probably resulting from higher cellularity compared to low grades.

In order to determine the optimal ROI for detecting tumor heterogeneity, we compared the diagnostic efficiency 
of the same parameters for different ROI. The inhomogeneity value derived from whole tumor showed better diagnostic performance than that from solid portions. For the whole tumor ROI, the sensitivity of inhomogeneity in glioma grading (HGGs vs. LGGs) was $94.4 \%$, indicating a high positive correlation. Hence, if the inhomogeneity is above 0.2162 , there is a high probability that the tumor will be a high grade. For solid portion ROI, the sensitivity of inhomogeneity in glioma grading (HGGs vs. LGGs) was $83.3 \%$, indicating a moderate positive correlation. The results of this study suggest that selecting the whole tumor as the ROI results in a better performance in evaluation of tumor heterogeneity. Because the whole tumor ROI contained all elements of tumor components, it provided the most accurate representation of glioma heterogeneity. Kang et al (22) demonstrated a sensitivity of $50 \%$ and a specificity of $100 \%$ in the differentiation of low and high grade gliomas with whole tumor volume analysis. Raja et al selected six consecutive slices from the centre of the tumour volume as ROI, and demonstrated the significance of DTI and DKI parameters in grading of glioma (29).

There are very few studies that have evaluated the peritumoral edema area, which theoretically correlates with the aggressiveness of the tumor. Guzman et al (30) reported that HGGs have higher ADC values in the peritumoral edema than LGGs. In our study, the mean and median ADC values for peritumoral edema did not differ significantly among patients with HGGs and LGGs in agreement with the other two authors $(31,32)$. Texture parameters such as entropy and the common value of edema volume, however, appeared to be helpful in distinguishing HGGs from LGGs. High grade gliomas tend to have a greater volume of edema and the extracellular water diffusion movement seemed to be more complex and disordered.

Ki-67, a nuclear antigen specific for proliferating cells (33), is used for the evaluation of tumor proliferation. The level of Ki-67 correlates with higher cell density and tumor grade for astrocytic gliomas (34). In previous studies DWI imaging and its estimate of ADC has been widely investigated, as it reflects tumor cellularity and proliferation (35). Some studies showed that the minimum or fifth percentile values of ADC correlated well with the Ki-67 labeling index. Our results demonstrated that the inhomogeneity value of ADC had a positive correlation with the $\mathrm{Ki}-67$ labeling index both in whole tumor and solid portion ROIs. The elevated Ki-67 labeling index correlated with tumor aggressiveness, and inhomogeneity reflected spatial irregularity and variability, these could be used to explain the positive relationship between inhomogeneity and the Ki-67 labeling index.

Apart from the intrinsic limits of any retrospective study, our study had several limitations. First, the number of cases analyzed was relatively small $(n=30)$ and thus larger studies are still necessary for further exploration. Second, we faced another challenge in determining the margin between the tumor and the edema, which can be indistinct, especially in low grade gliomas. Additionally, the differentiations between edema and tumor infiltration and between the nonenhancing solid portion of the tumor and microcystic necrosis are impossible via visual inspection because microcystic necrosis and nonenhancing solid portions showed intermediate signal intensity on T2WI and hypointensity on CE T1WI (36). Third, the measurements of tumor heterogeneity in our study were performed by a single blinded operator which potentially could introduce bias. Further studies should evaluate the inter-operator and intra-operator variability, which is a limitation in this study. Fourth, we used the manual segmentation method to define tumor boundaries, which lacks reproducibility. Thus we can use multi-sequence fused imaging to draw the outline of tumor boundaries.

In conclusion, our results suggested that texture analysis of DWI based on different ROI can provide various significant parameters to evaluate tumor heterogeneity, which was correlated with tumor grade. Particularly, the inhomogeneity value obtained from whole tumor ROI provided high diagnostic value in differentiating HGGs from LGGs. Moreover, texture analysis of DWI will be helpful to get the expression status of some biomarkers such as $\mathrm{Ki}-67$ labeling index via the non-invasive and objective methods.

\section{Acknowledgements}

Not applicable.

\section{Funding}

The present study was supported by National Natural Science Foundation of China (grant no. 81771904).

\section{Availability of data and materials}

The datasets used and/or analyzed during the current study are available from the corresponding author on reasonable request.

\section{Authors' contributions}

KX and SW conceived and designed the study. MM, XZ, CW, RW and MUS collected and processed the data. JFW provided the technique methods of texture analysis. KX, SW and MM wrote the paper. KX, SW, MM, XZ and MUS reviewed and edited the manuscript. All authors read and approved the final manuscript.

\section{Ethics approval and consent to participate}

The present study was approved by the Ethical Committee of the First Affiliated Hospital of Xuzhou Medical University, Xuzhou, China (approval number XYFY2017-KL003-01). All patients provided written informed consent for participation in the present study.

\section{Consent for publication}

Not applicable.

\section{Competing interests}

The authors declare that they have no competing interests.

\section{References}

1. Louis DN, Ohgaki H, Wiestler OD, Cavenee WK, Burger PC Jouvet A, Scheithauer BW and Kleihues P: The 2007 WHO classification of tumours of the central nervous system. Acta Neuropahtol 114: 97-109, 2007. 
2. Skogen K, Ganeshan B, Good C, Critchley G and Miles K: Measurements of heterogeneity in gliomas on computed tomography relationship to tumour grade. J Neurooncol 111: 213-219, 2013.

3. Weller M: Novel diagnostic and therapeutic approaches to malignant glioma. Swiss Med Wkly 141: w13210, 2011.

4. Server A, Kulle B, Gadmar OB, Josefsen R, Kumar T and Nakstad PH: Measurements of diagnostic examination performance using quantitative apparent diffusion coefficient and proton MR spectroscopic imaging in the preoperative evaluation of tumor grade in cerebral gliomas. Eur J Radiol 80: 462-470, 2011.

5. Chenevert TL, Stegman LD, Taylor JM, Robertson PL, Greenberg HS, Rehemtulla A and Ross BD: Diffusion magnetic resonance imaging: An earlysurrogate marker of therapeutic efficacy in brain tumors. J Natl Cancer Inst 92: 2029-2036, 2000

6. Ellingson BM, Cloughesy TF, Zaw T, Lai A, Nghiemphu PL Harris R, Lalezari S, Wagle N, Naeini KM, Carrillo J, et al: Functional diffusion maps (fDMs) evaluated beforeand after radiochemotherapy predict progression-free and overall survival in newly diagnosed glioblastoma. Neuro Oncol 14: 333-343, 2012.

7. Ellingson BM, Cloughesy TF, Lai A, Nghiemphu PL, Liau LM and Pope WB: Quantitative probabilistic functional diffusion mapping in newly diagnosed glioblastoma treated with radiochemotherapy. Neuro Oncol 15: 382-390, 2013.

8. Rowley HA, Grant PE and Roberts TP: Diffusion MR imaging. Theory and applications. Neuroimaging Clin N Am 9: 343-361, 1999.

9. Sugahara T, Korogi Y, Kochi M, Ikushima I, Shigematu Y, Hirai T, Okuda T, Liang L, Ge Y, Komohara Y, et al: Usefulness of diffusion-weighted MRI with echo-planar technique in the evaluation of cellularity in gliomas. J Magn Reson Imaging 9: 53-60, 1999.

10. Ellingson BM, Malkin MG, Rand SD, Connelly JM, Quinsey C, LaViolette PS, Bedekar DP and Schmainda KM: Validation of functional diffusion maps (fDMs) as a biomarker for human glioma cellularity. J Magn Reson Imaging 31: 538-548, 2010.

11. Nie K, Chen JH, Yu HJ, Chu Y, Nalcioglu O and Su MY: Quantitative analysis of lesion morphology and texture features for diagnostic prediction in breast MRI. Acad Radiol 15 $1513-1525,2008$

12. Georgiadis P, Cavouras D, Kalatzis I, Glotsos D, Athanasiadis E, Kostopoulos S, Sifaki K, Malamas M, Nikiforidis G and Solomou E: Enhancing the discrimination accuracy between metastases, gliomas and meningiomas on brain MRI by volumetric textural features and ensemble pattern recognition methods. Magn Reson Imaging 27: 120-130, 2009.

13. Chicklore S, Goh V, Siddique M, Roy A, Marsden PK and Cook GJ: Quantifying tumour heterogeneity in 18F-FDGPET/CT imaging by texture analysis. Eur J Nucl Med Mol Imaging 40: 133-140, 2013

14. Assefa D, Keller H, Ménard C, Laperriere N, Ferrari RJ and Yeung I: Texture features for response monitoring of glioblastoma multiforme on T1-weighted and T2-FLAIR MR images: A preliminary investigation in terms of identification and segmentation. Med Phys 37: 1722-1736, 2010.

15. Herlidou-Même S, Constans JM, Carsin B, Olivie D, Eliat PA, Nadal-Desbarats L, Gondry C, Le Rumeur E, Idy-Peretti I and de Certaines JD: MRI texture analysis on texture test objects, normal brain and intracranial tumors. Magn Reson Imaging 21: 989-993, 2003

16. Zacharaki EI, Wang S, Chawla S, Soo Yoo D, Wolf R, Melhem ER and Davatzikos C: Classification of brain tumor type and grade using MRI texture and shape in a machine learning scheme, Magn Reson Med 62: 1609-1618, 2009.

17. Li H, Giger ML, Lan L, Bancroft Brown J, MacMahon A, Mussman M, Olopade OI and Sennett C: Computerized analysis of mammographic parenchymal patterns on a large clinical dataset of full-field digital mammograms: Robustness study with two high-risk datasets. J Digit Imaging 25 591-598, 2012

18. Brown R, Zlatescu M, Sijben A, Roldan G, Easaw J, Forsyth P, Parney I, Sevick R, Yan E, Demetrick D, et al: The use of magnetic resonance imaging to noninvasively detect genetic signatures in oligodendroglioma. Clin Cancer Res 14: 2357-2362, 2008.
19. Nilsson J, Holgersson G, Carlsson T, Henriksson R, Bergström S and Bergqvist $\mathrm{M}$ : Incidence trends in high-grade primary brain tumors in males and females. Oncol Lett 13: 2831-2837, 2017.

20. Kang H, Lee HY, Lee KS and Kim JH: Imaging-based tumor treatment response evaluation: Review of conventional, new and emerging concepts. Korean J Radiol 13: 371-390, 2012.

21. Skogen K, Schulz A, Dormagen JB, Ganeshan B, Helseth E and Server A: Diagnostic performance of texture analysis on MRI in grading cerebral gliomas. Eur J Radiol 85: 824-829, 2016.

22. Kang Y, Choi SH, Kim YJ, Kim KG, Sohn CH, Kim JH, Yun TJ and Chang KH: Gliomas: Histogram analysis of apparent diffusion coefficient maps with standard- or high-b-value diffusion-weighted MR imaging-correlation with tumor grade. Radiology 261: 882-890, 2011.

23. Emblem KE, Nedregaard B, Nome T, Due-Tonnessen $P$, Hald JK, Scheie D, Borota OC, Cvancarova M and Bjornerud A: Glioma grading by using histogram analysis of blood volume heterogeneity from MR-derived cerebral blood volume maps. Radiology 247: 808-817, 2008.

24. Fan GG, Deng QL, Wu ZH and Guo QY: Usefulness of diffusion/perfusion-weighted MRI in patients with non-enhancing supratentorial brain gliomas: Avaluable tool to predict tumour grading? Br J Radiol 79: 652-658, 2006.

25. Maia AC Jr, Malheiros SM, da Rocha AJ, da Silva CJ, Gabbai AA and Ferraz FA, Stávale JN: MR cerebral blood volume maps correlated with vascular endothelial growth factor expression and tumor grade in nonenhancing gliomas. AJNR Am J Neuroradiol 26: 777-783, 2005

26. Ryu YJ, Choi SH, Park SJ, Yun TJ and Kim JH: Glioma: Application of whole-tumor texture analysis of diffusion-weighted imaging for the evaluation of tumor heterogeneity. PLoS One 9: e108335, 2014.

27. Davnall F, Yip CS, Ljungqvist G, Selmi M, Ng F, Sanghera B, Ganeshan B, Miles KA, Cook GJ and Goh V: Assessment of tumor heterogeneity: An emerging imaging tool for clinical practice? Insights Imaging 3: 573-589, 2012.

28. Yang D, Korogi Y, Sugahara T, Kitajima M, Shigematsu Y, Liang L, Ushio Y and Takahashi M: Cerebral gliomas: Prospective comparison of multivoxel 2D chemical-shift protonMR spectroscopy, echoplanar perfusion and diffusion-weighted MRI. Neuroradiology 44: 656-666, 2002.

29. Raja R, Sinha N, Saini J, Mahadevan A, Rao KN and Swaminathan A: Assessment of tissue heterogeneity using diffusion tensor and diffusion kurtosis imaging for grading gliomas. Neuroradiology 58: 1217-1231, 2016.

30. Guzman R, Altrichter S, El-Koussy M, Gralla J, Weis J, Barth A, Seiler RW, Schroth G and Lövblad KO: Contribution of the apparent diffusion coefficient in perilesional edema for the assessment of brain tumors. J Neuroradiol 35: 224-229, 2008.

31. Castillo M, Smith JK, Kwock L and Wilber K: Apparent diffusion coefficients in the evaluation of high-grade cerebral gliomas. Am J Neuroradiol 22: 60-64, 2001.

32. Guo AC, Cummings TJ, Dash RC and Provenzale JM: Lymphomas and high-grade astrocytomas: Comparison of water diffusibility and histologic characteristics. Radiology 224: 177-183. 2002

33. Scholzen T and Gerdes J: The Ki-67 protein: From the known and the unknown. J Cell Physiol 182: 311-322, 2000.

34. Kiss R, Dewitte O, Decaestecker C, Camby I, Gordower L, Delbecque K, Pasteels JL, Brotchi J and Salmon I: The combined determination of proliferative activity and cell density in the prognosis of adult patients with supratentorial high-grade astrocytic tumors. Am J Clin Pathol 107: 321-331, 1997.

35. Brynolfsson P, Nilsson D, Henriksson R, Hauksson J and Karlsson M: ADC texture-An imaging biomarker for high-grade glioma? Med Phys 41: 101903, 2014.

36. Fan GG, Deng QL, Wu ZH and Guo QY: Usefulness of diffusion/perfusion weighted MRI in patients with non-enhancing supratentorial brain gliomas: A valuable tool to predict tumour grading? Br J Radiol 79: 652-658, 2006. 